\title{
3D-2D-3D photonic crystal heterostructures fabricated by direct laser writing
}

\author{
M. Deubel and M. Wegener \\ Institut für Angewandte Physik, Universität Karlsruhe (TH), Wolfgang-Gaede-Straße 1, D-76131 Karlsruhe, Germany \\ S. Linden and G. von Freymann \\ Institut für Nanotechnologie, Forschungszentrum Karlsruhe in der Helmholtz-Gemeinschaft, Postfach 3640, \\ D-76021 Karlsruhe, Germany \\ S. John \\ Department of Physics, University of Toronto, 60 St. George Street, Toronto, Ontario M5S 1A7, Canada
}

Received October 25, 2005; accepted November 22, 2005; posted December 5, 2005 (Doc. ID 65578)

Using direct laser writing, we fabricate photoresist templates for 3D-2D-3D photonic crystal heterostructures for what we believe to be the first time. The optical properties of these structures are directly compared with the theoretical ideal, revealing good agreement and hence good sample quality. This provides an experimental starting point for the microfabrication and testing of broadband, 3D air-waveguide microcircuitry in photonic bandgap materials. (C) 2006 Optical Society of America

OCIS codes: $220.4000,160.5470,300.6340$.

The quest for the ever denser integration of functional components in planar electronic circuits drives nanotechnology to reduce lateral feature sizes (Moore's law). To some extent today's electronics also escapes into the third dimension by using many layers of interconnection on a $2 \mathrm{D}$ chip. Truly $3 \mathrm{D}$ electronic chip circuitry, however, is currently not in sight because of prohibitive heat generation and electrical cross talk.

For optical circuits, which are admittedly far less developed than their electronic counterparts, the wavelength of light sets a fundamental limit regarding lateral integration density. This limit can almost be reached in 2D slab waveguide photonic crystal circuits. ${ }^{1,2}$ Here radiation losses into the third dimension pose fundamental performance limitations. It has been argued that these limitations can be overcome in 3D-2D-3D photonic crystal heterostructures ${ }^{3-6}$ in which a 2 D photonic crystal layer (comprising waveguides, etc.) is clad by $3 \mathrm{D}$ photonic bandgap materials on both sides (Fig. 1). In principle this approach totally eliminates losses into the third dimension for frequencies in the 3D photonic bandgap. Furthermore, it allows the incorporation of several parallel 2D optical microchip layers separated by a few optical wavelengths. Architectures have been proposed theoretically that even allow broadband interconnecting waveguide structures between the different planes, leading to a true 3D photonic circuitry.

These recent concepts have not yet reached the proof-of-principle stage in materials synthesis. Here we take the first significant steps by using direct laser writing (DLW) for the fabrication of corresponding photoresist templates. Combined with the recent breakthrough of silicon double inversion of polymeric templates 8 leading to the required high-index contrast structures, our work provides a starting point for realizing the above far-reaching goals.
It is well known that DLW essentially allows the fabrication of practically arbitrary 3D photoresist structures. ${ }^{9-11}$ 3D photonic crystals with stop bands at telecommunications frequencies, e.g., wood piles $^{12,13}$ and slanted-pore structures, ${ }^{14}$ of superb quality have recently been demonstrated and characterized optically. To evaluate the question of how far one gets regarding 3D-2D-3D photonic crystal heterostructures we have performed two different sets of experiments [(i) and (ii) (Fig. 1)]. In set (i) we have fabricated 3D woodpile photonic crystals and have added a 2D photonic crystal plane including waveguides on top. Here, we deliberately do not add the top 3D photonic crystal in order to be able to inspect the fabricated structures by using highresolution electron microscopy. In set (ii) (corresponding to the background of Fig. 1) we add the top 3D

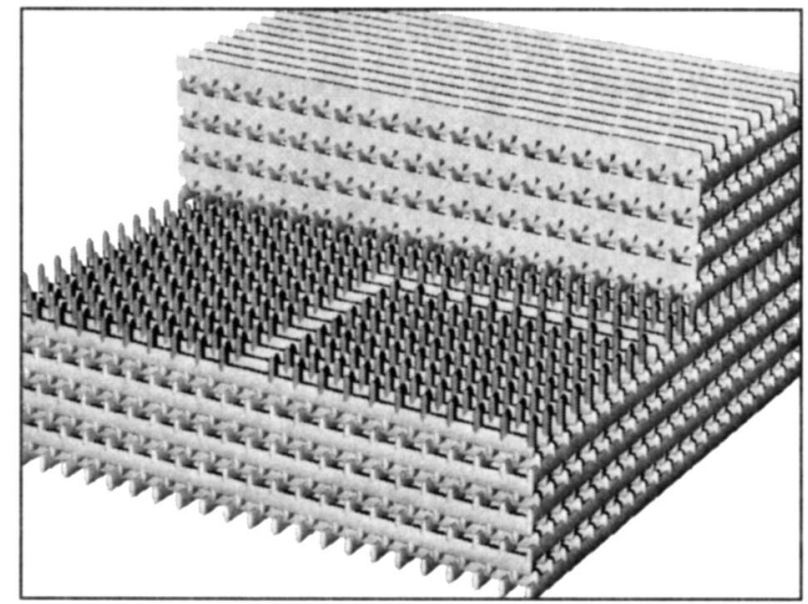

Fig. 1. Scheme of a 3D-2D-3D photonic crystal heterostructure. The foreground corresponds to the first set of experiments (i) presented in Fig. 2, and the background corresponds to the second set of experiments (ii) shown in Fig. 3. 

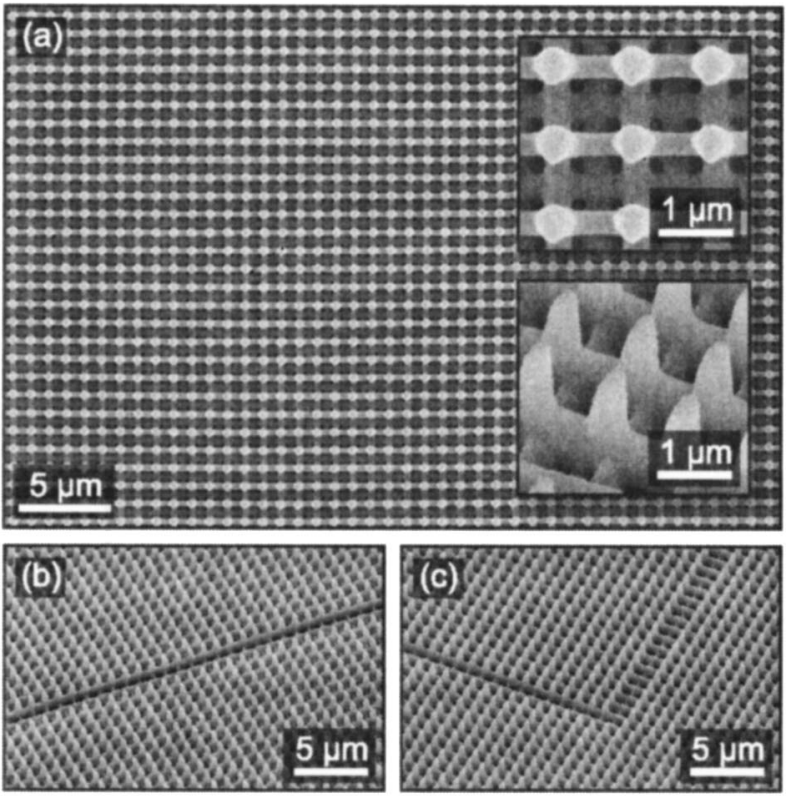

Fig. 2. (a)-(c) Gallery of different 3D-2D photonic crystal heterostructures comprising waveguide structures, all fabricated by DLW. The top 3D photonic crystal has deliberately not been written (compare Fig. 1). Note the good relative alignment of the $3 \mathrm{D}$ and the $2 \mathrm{D}$ photonic crystal.

structure. Such samples can obviously no longer be easily characterized by electron microscopy, but they can be characterized by optical spectroscopy. It is clear that for incidence of light normal to the 2D plane, the structure is just a Fabry-Perot cavity. The corresponding spectra can then be directly compared with spectra obtained from (numerically exact) scattering-matrix calculations. Obviously, in-plane spectroscopy would become possible only after silicon double inversion, which is beyond the scope of this work.

In multiphoton $\mathrm{DLW}^{9-11,13,14}$ femtosecond laser pulses are tightly focused into a photoresist that exhibits negligible one-photon absorption at the wavelength of the laser light. However, inside a small volume element (a voxel) in the focus, the photoresist can be exposed by multiphoton absorption. After development only the exposed regions remain. By scanning the photoresist in three dimensions relative to the fixed focus, essentially arbitrary 3D structures can be fabricated. Using a focusing lens with a numerical aperture $\mathrm{NA}=1.4$, and the commercially available photoresist SU-8, we routinely achieve voxel sizes down to a $150 \mathrm{~nm}$ lateral diameter. All the details of our DLW setup and the SU-8 process parameters can be found in Ref. 13. All structures are surrounded by a massive wall ${ }^{13}$ to reduce the effects of strain due to photoresist shrinkage during development.

Figure 2 shows an electron micrograph gallery of 3D-2D photonic crystal heterostructures fabricated by DLW. The bottom 3D photonic crystal is a woodpile $^{12}$ with a rod spacing of $a=1 \mu \mathrm{m}$, a lattice constant of $c=\sqrt{2} a, 16$ layers, and an $80 \mu \mathrm{m} \times 80 \mu \mathrm{m}$ area. In this first set of experiments, (i), the top 3D photonic crystal has deliberately not been written to allow for inspection of the $2 \mathrm{D}$ plane. It is apparent that the relative alignment between the $3 \mathrm{D}$ and the $2 \mathrm{D}$ structure is very good even though we have used a fast-scan mode for the 3D structure (Ref. 13) and a shot-by-shot mode for the 2D structure. The shot-byshot mode allows us to control the size of each and every dielectric post in the 2D plane independently, thus allowing for arbitrary waveguide structures. The structures exemplified here are not necessarily optimized or optically functional for waveguiding but rather aim at a proof of principle. The writing time for the structure shown in Fig. 2, for example, is about $20 \mathrm{~min}$.

To assess the optical quality of such structures we fabricated a second set of samples, (ii), with a $2 \mathrm{D}$ photonic crystal plane identical to the one shown in Fig. 2 (without waveguides) and with a $3 \mathrm{D}$ woodpile structure added on the top. The parameters of this woodpile are identical to those at the bottom (both 16 layers). The height of the $2 \mathrm{D}$ photonic crystal is $150 \mathrm{~nm}$. Normal or oblique incidence transmittance spectroscopy is expected to reveal a peak related to the Fabry-Perot mode of the $2 \mathrm{D}$ structure clad between two 3D photonic crystal mirrors, provided that this mode lies in the fundamental stop
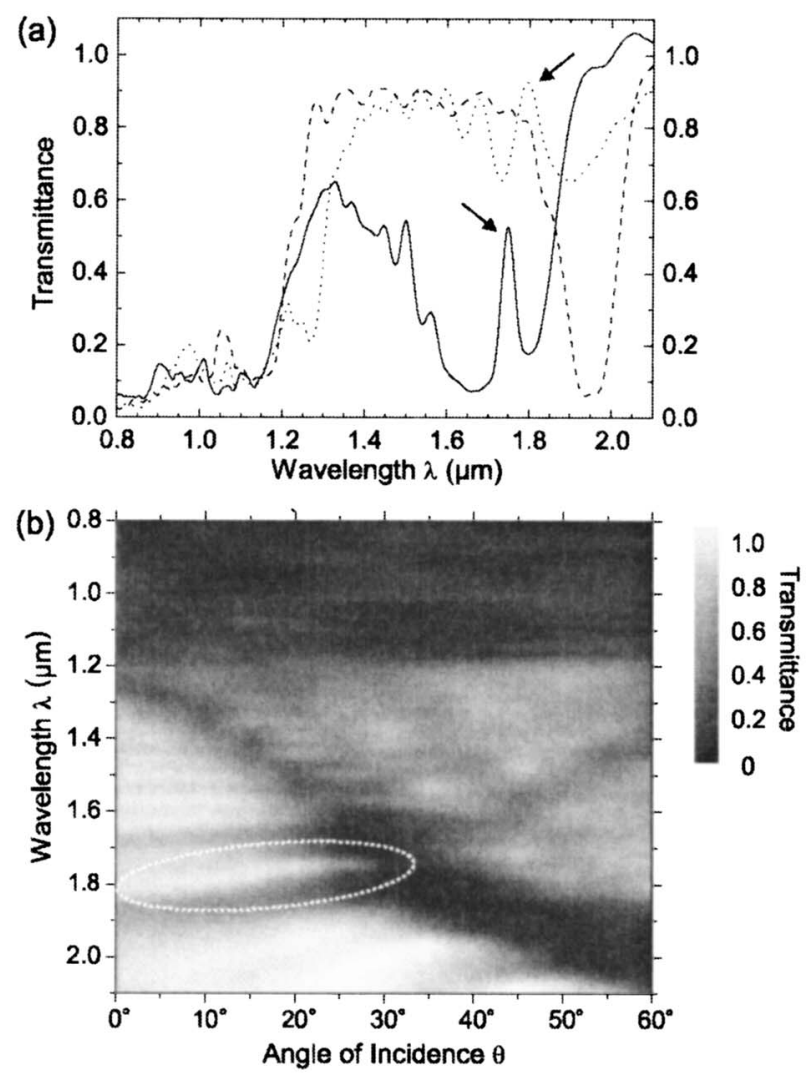

Fig. 3. Optical transmittance spectra of a structure similar to those in Fig. 2, but without waveguides and with a top 3D woodpile photonic crystal added (compare Fig. 1). (a) Selected individual spectra: $\theta=0^{\circ}, p$ polarization (dotted curve); $\theta=25^{\circ}, s$ polarization (solid curve); $\theta=50^{\circ}, p$ polarization (dashed curve). (b) Gray-scale plot of the transmittance versus angle and wavelength for $s$ polarization. The arrows in (a) and the dotted ellipse in (b) mark the spectral features associated with the $2 \mathrm{D}$ photonic crystal layer. 

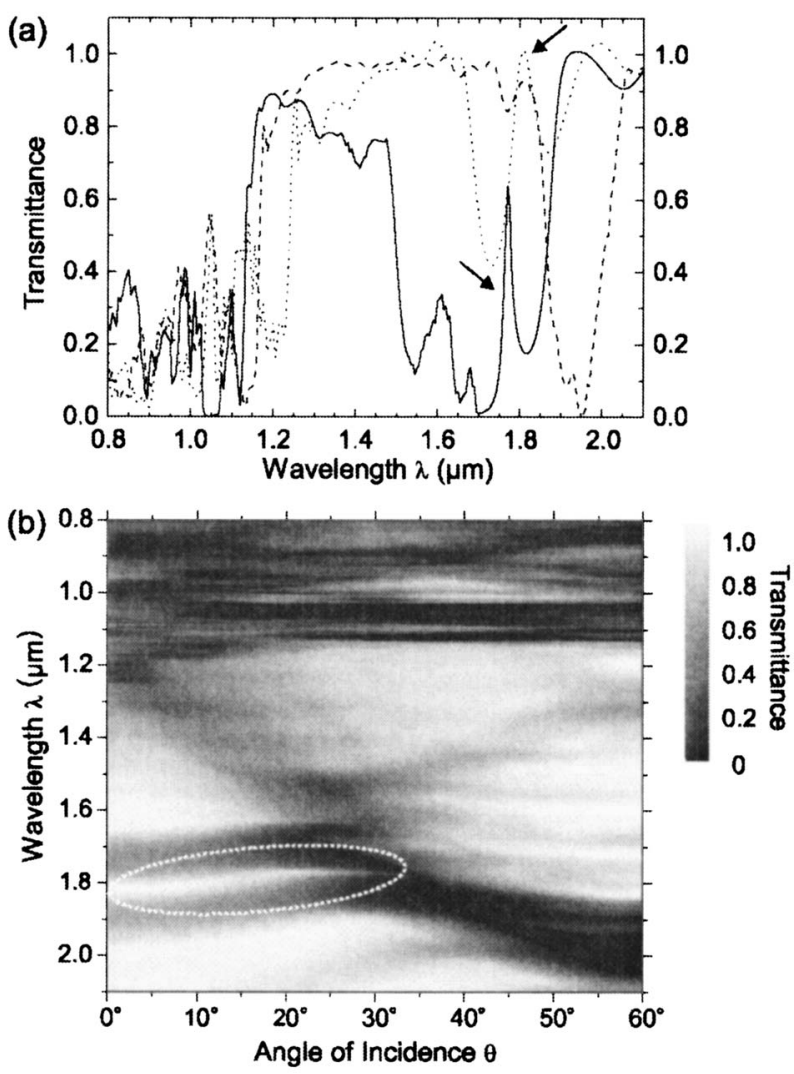

Fig. 4. Calculated transmittance spectra corresponding to the structure and the parameters of the experiment (Fig. 3 ). Note the good overall agreement with the experiment.

band of the woodpile, a condition that can be controlled by the angle of incidence $\theta$ with respect to the surface normal [see the dotted ellipse in Fig. 3(b)]. Figure 3(a) shows corresponding individual measured spectra taken with a home-built setup that has an opening angle of the light impinging on the sample of approximately $\pm 5^{\circ}$ and at the same time allows spectroscopy on small samples by introducing an aperture in an intermediate image plane. Here we effectively image a sample area of approximately $[30 \mu \mathrm{m} / \cos (\theta)] \times 40 \mu \mathrm{m}$. To ensure reliable and reproducible results the spectra are referenced to the bare glass substrate for each angle $\theta$ and polarization, which implies that the transmittance defined this way can slightly exceed unity at angles and wavelengths where the photonic crystal acts as an effective antireflection coating of the glass substrate. The spectra in Fig. 3 reveal a stop band in transmittance related to the 3D woodpile structure very closely similar to the data presented in Ref. 13. In or close to that stop band, an additional peak associated with the $2 \mathrm{D}$ photonic crystal plane occurs [the arrows in Fig. 3(a) and the dotted ellipse in Fig. 3(b)].

While this qualitative behavior is expected intuitively (see above), it is not immediately clear how close these spectra come to the theoretical ideal. Thus we compare the measured spectra directly with theory by computing the transmittance spectra by using a scattering-matrix approach ${ }^{15}$ for different angles. For the calculations the ellipsoidal shape of the voxels is taken into account. The SU-8 photore- sist has a real refractive index of $n=1.57$, and its surface roughness is neglected. To mimic the finite opening angle of the experimental apparatus (see above) for a direct comparison, we average the calculated spectra over a range of $\pm 5^{\circ}$. Furthermore the transmittance is defined in the same way as in the experiment. The corresponding results are depicted in Fig. 4 and are represented in the same way as those in the experiment in Fig. 3. The clear, overall agreement between theory and experiment is a strong indication of the high quality of the $3 \mathrm{D}-2 \mathrm{D}-3 \mathrm{D}$ heterostructure.

In conclusion, we have fabricated what we believe to be the first 3D-2D-3D photonic crystal heterostructures. The structures made by direct laser writing are characterized by optical spectroscopy. The comparison of these data with theory reveals good agreement, hence good quality of the fabricated structures. If replicated in silicon-a crucial step that has very recently been demonstrated by using a double-inversion procedure-such structures might allow for 3D air-waveguide photonic bandgap microcircuitry, as suggested by theory.

We acknowledge support by the Deutsche Forschungsgemeinschaft and the State of BadenWürttemberg through subproject A1.4 of the DFGForschungszentrum "Functional Nanostructures" (CFN) and by DFG projects We 1497/9-1 and Fr 1671/ 4-3. S. John acknowledges the support of the Natural Sciences and Engineering Research Council of Canada. M. Deubel's e-mail address is markus.deubel@physik.uni-karlsruhe.de.

\section{References}

1. J. D. Joannopoulos, P. R. Villeneuve, and S. Fan, Nature 386, 143 (1997).

2. S. Noda, A. Chutinan, and M. Imada, Nature 407, 608 (2000)

3. E. Istrate, M. Charbonneau-Lefort, and E. H. Sargent, Phys. Rev. B 66, 075121 (2002).

4. A. Chutinan, S. John, and O. Toader, Phys. Rev. Lett. 90, 123901 (2003).

5. A. Chutinan and S. John, Photonics Nanostruct. Fundam. Appl. 2, 41 (2004).

6. A. Chutinan and S. John, Phys. Rev. E 71, 026605 (2005).

7. A. Chutinan and S. John, Phys. Rev. B 72, 161316 (2005)

8. N. Tétreault, G. von Freymann, M. Deubel, M. Hermatschweiler, F. Pérez-Willard, S. John, M. Wegener, and G. A. Ozin, Adv. Mater. (Weinheim, Ger.) (to be published), DOI 10.1002/adma. 200501674 .

9. H.-B. Sun, S. Matsuo, and H. Misawa, Appl. Phys. Lett. 74, 786 (1999).

10. S. Kawata, H.-B. Sun, T. Tanaka, and K. Takada, Nature 412, 697 (2001).

11. M. Straub and M. Gu, Opt. Lett. 27, 1824 (2002).

12. K. M. Ho, C. T. Chan, C. M. Soukoulis, R. Biswas, and M. Sigalas, Solid State Commun. 89, 413 (1994).

13. M. Deubel, G. von Freymann, M. Wegener, S. Pereira, K. Busch, and C. M. Soukoulis, Nat. Mater. 3, 444 (2004).

14. M. Deubel, M. Wegener, A. Kaso, and S. John, Appl. Phys. Lett. 85, 1895 (2004).

15. D. M. Whittaker and I. S. Culshaw, Phys. Rev. B 60, 2610 (1999). 